hep-lat/9712012

\title{
Tempered Fermions in the Hybrid Monte Carlo Algorithm
}

\author{
G. BOYD*a \\ ${ }^{a}$ Centre for Computational Physics, University of Tsukuba, Tsukuba, Ibaraki 305, Japan. \\ email:boyd rccp.tsukuba.ac.jp
}

Parallel tempering simulates at many quark masses simultaneously, by changing the mass during the simulation while remaining in equilibrium. The algorithm is faster than pure HMC if more than one mass is needed, and works better the smaller the smallest mass is.

\section{INTRODUCTION}

The standard algorithms used for full QCD are painfully slow. Physically large objects, like instantons, pions etc., may well decorrelate much more slowly than, say, the plaquette [1].

Tempered algorithms [1 3] promote a parameter of the theory to a dynamical variable that changes during the simulation, which has tremendous potential for speeding up slow simulations. They have been successfully implemented in $\beta$ for spin glasses, U(1) etc. For QCD at zero temperature promoting $\beta$ does not help (although at high temperature, around the chiral phase transition it probably will), but promoting the quark mass, and allowing it to change during the simulation, does speed things up.

The mass is only changed if the configuration is simultaneously in the equilibrium distribution of both masses. So tempering is always in equilibrium and requires no re-weighting etc. afterwards.

The minimum gain comes from running at heavier (faster) masses between independent configurations. The maximum gain comes if the relevant auto-correlations are smaller for larger masses.

\footnotetext{
${ }^{*}$ Combined proceedings for Lattice 97, Edinburgh and the International Workshop 'Lattice QCD on Parallel Computers', University of Tsukuba, Japan.
}

\section{TEMPERING}

The quark mass, $a m_{\mathrm{q}}$ for staggered or $\kappa$ for Wilson fermions, becomes a dynamic variable, and may take a different value for each trajectory of, say, the hybrid Monte Carlo algorithm [4]. (Regard $m$ below as the corresponding $\kappa$ for Wilson fermions.) The masses used belong to an ordered set with $N_{m}$ elements, $\left[m_{\min }, \ldots, m_{\max }\right]$. The only requirement is that the action histograms of neighbouring masses overlap.

There are two types of tempering, simulated and parallel tempering. The idea behind simulated tempering in QCD, investigated in [3], is very simple, and as it is the basis for the parallel tempering investigated here it will be described first.

In simulated tempering you add to the probability distribution a constant $g_{i}$ for each mass $m_{i}$, which indicates roughly where the half-way point between the action histograms of mass $m_{i}$ and $m_{i+1}$ is. The original QCD probability distribution $P(U, \phi)$ now becomes

$$
P(U, \phi, i) \propto \exp \left[-S\left(U, \phi, \beta, m_{i}\right)+g_{i}\right] .
$$

This distribution is simulated using your favourite algorithm for fixed quark mass (eg., HMC here), combined with Metropolis steps to change from $m_{i}$ to $m_{i \pm 1}$. The constants $g_{i}$ are only to enable the masses to change both up and down, and do not affect the physics.

The hybrid Monte Carlo algorithm insures that the correct Gibbs distribution is generated at each value of the mass, and the temper- 
ing Metropolis step insures that the mass only changes if the configuration is part of the equilibrium distribution of both masses.

The constants $g_{i}$ can be chosen freely, depending on what seems best for the simulation. They do not affect the physics, only the frequency with which each mass is visited. The $g_{i}$ can be fixed by choosing, for example, to visit each mass with equal probability, $P(i)=1 / N_{m}$. Then $g_{i}=-\ln Z_{i}$, ie. the original free energy at fixed mass $m_{i}$. The choice is arbitrary, though, and can be optimized for speed.

The simulation only needs $g_{i+1}-g_{i}$, and a good starting point is to take the first two terms below:

$$
\Delta g=-\langle\bar{\psi} \psi\rangle V \delta m-\langle\chi\rangle V(\delta m)^{2}+O\left((\delta m)^{3}\right)
$$

where $\langle\bar{\psi} \psi\rangle$ and $\langle\chi\rangle$ are the chiral condensate and susceptibility. The requirement of overlapping histograms implies that $\delta m$ satisfies

$$
\delta m \sim 1 / \sqrt{\langle\chi\rangle V} \sim m_{\sigma} / \sqrt{V} .
$$

The overhead depends on the step size $\delta m$. As the susceptibility is related to the scalar meson mass, $\chi=A_{\sigma} / m_{\sigma}^{2}$, the step size is large in the chiral limit. Hence simulated tempering becomes more effective for very small quark masses, with the gain in speed more than compensating for the $N_{m}^{2}$ cost of having additional masses.

The volume dependence above is in units of some relevant correlation length, so taking the continuum limit in fixed physical volume does not cause the method to break down.

A further improvement comes if you temper in a parallel way. If $N_{m}$ different masses are needed, you can happily do $N_{m}$ different simulated tempering runs simultaneously on different computers. You can even go one better, and put them together on one computer in a way that removes the need for the constants $g_{i}$ and improves the performance. This is called parallel tempering; results are presented in the next section.

In parallel tempering you first generate one (thermalised) configuration $C_{i}$ at each of the masses. Then use a Metropolis step to decide whether the configuration $C_{i}$ at mass $m_{i}$, and configuration $C_{i+1}$ at mass $m_{i+1}$ should be swapped for the next trajectory. If this is done,
Table 1

Parameters and results from the tempered (T) and a standard HMC run. The trajectories have unit length. The last three columns give the acceptance rate for the Metropolis mass changes, and the integrated autocorrelation times of the plaquette and $2 \times 2$ Wilson loop.

\begin{tabular}{rrrrl}
\hline \hline$m$ & $\mathrm{~s} /$ trj & $\%$ & $\tau_{\text {int }}^{P}$ & $\tau_{\text {int }}^{W 22}$ \\
\hline (T) 0.020 & 475 & 15 & $4.9(14)$ & $3.6(10)$ \\
(T) 0.024 & 350 & 21 & $3.3(8)$ & $2.8(6)$ \\
(T) 0.028 & 283 & 24 & $4.2(15)$ & $3.3(10)$ \\
(T) 0.032 & 231 & 21 & $3.5(9)$ & $2.7(10)$ \\
(T) 0.036 & 193 & - & $3.6(12)$ & $2.8(6)$ \\
(HMC) 0.020 & 475 & - & $9.0(21)$ & $8.4(17)$ \\
(HMC) 0.040 & 166 & - & $8.5(20)$ & $6.9(15)$ \\
(HMC) 0.060 & 84 & - & $7.3(13)$ & $5.8(13)$ \\
\hline \hline
\end{tabular}

the next trajectory will run with $C_{i+1}$ at mass $m_{i}$, and $C_{i}$ at mass $m_{i+1}$. After each trajectory one starts trying to swap masses 1 and 2 , moving up through the list, ending by trying to swap the configurations at masses $N_{m}-1$ and $N_{m}$.

This method doesn't need any constants $g_{i}$, changes the mass at two rather than one configuration, and has the further advantage of generating a configuration at each mass every trajectory. Also, trajectories way out in the tail of the distribution stand a chance of moving more than one step in mass. This doesn't happen very often though!

\section{RESULTS}

Two sets of runs are in progress with four staggered fermions on an $8^{3} \times 12$ lattice. The tempered one has five masses, $\{0.020,0.024,0.028,0.032,0.036\}$, called set ' $\mathrm{T}$ '. For comparison there are three standard HMC runs at masses $0.020,0.040$ and 0.060 . The run parameters are given in table 1. So far about 2000 units in $\tau$ have been run for $\mathrm{T}$, and about 3500 for the HMC run.

Which of the five configurations of the tempered run used $m=0.020$ at which time can be 


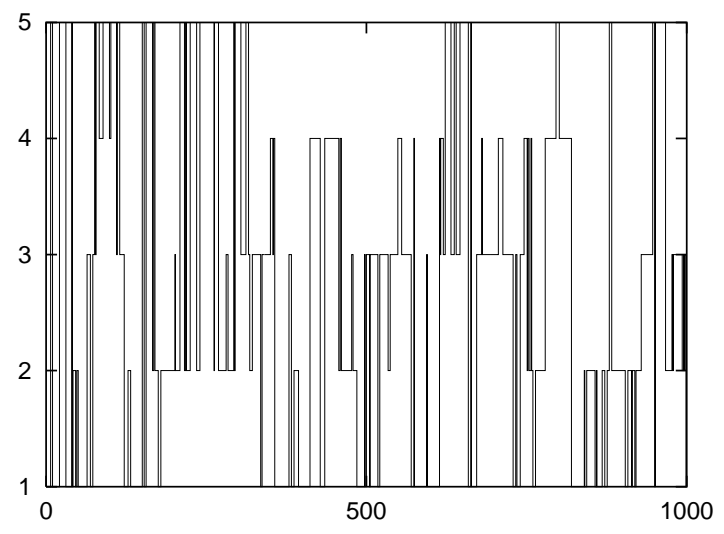

Figure 1. Part of the time history of the configuration number running at $m=0.020$ in the tempered run.

seen in the time history of figure 1. It is clear that all five configurations have run at each mass about equally often, as required.

The acceptance rate for transitions between masses is also shown in table 11, and lies around 20\%. Another run with closer masses, $\{0.020,0.023,0.026,0.029,0.032\}$, yielded rates about ten percentage points higher. An acceptance rate of around $20 \%$ to $30 \%$ seems optimal.

A measure of the speed of an algorithm is $\tau_{\text {int }}^{O}$, the integrated auto-correlation [5] for an observable $O$. With insufficient data, $N_{\text {data }}<1000 \tau_{\text {int }}$, an accurate value cannot be obtained. The largest value for $\tau_{\text {int }}^{O}$ of all observables $O$ defines the number of independent configurations.

For full QCD the global topological charge $Q$ seems to be the slowest observable[1] . However, on this size lattice the topology (field theoretic definition) turned out to depend on the action used for cooling, and is probably not well defined?. The plaquette and Wilson loops up to $2 \times 2$ seem to have the most well defined auto-

${ }^{2}$ The cooling actions tested used $1 \times 1$ and $1 \times 2$ loops with various values of the coefficients. On a given configuration different choices for the action lead to completely different global topological charges.

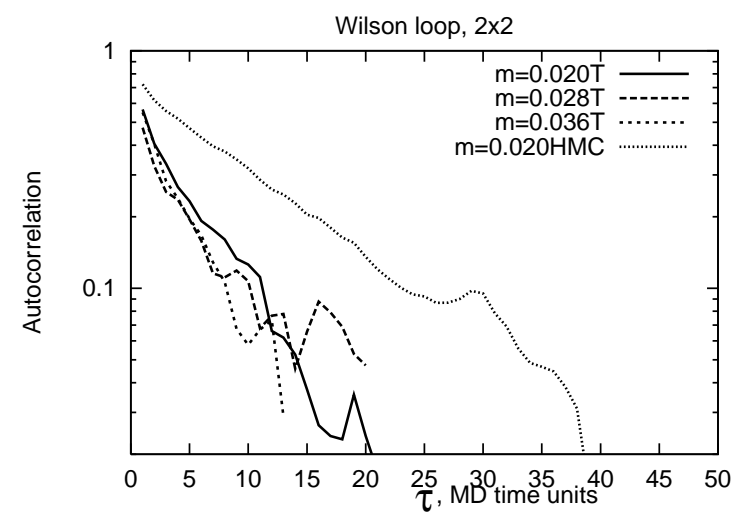

Figure 2. The auto-correlation function for the $2 \times 2$ Wilson loop at $m=0.020$ from set $\mathrm{T}$ and a standard HMC reference run.

correlation, and have been used here.

In figure 2 the auto-correlation function for the $2 \times 2$ Wilson loop at $m=0.020$ from the tempering and a standard HMC run is plotted. The integrated autocorrelations obtained for both the plaquette and the $2 \times 2$ Wilson loop are given in table 1. These turn out to be about $20 \%$ smaller than the slope parameter needed to fit the central part of the correlator in figure 2 to an exponential.

For observables from the tempered run, $\tau_{\text {int }}$ is about three times smaller than from the standard HMC run. The computer time needed per tempered trajectory, $T_{\mathrm{T}}$, compared with the time for a single HMC run at the smallest mass yields $T_{\mathrm{T}}=3.22 T_{\mathrm{HMC}}^{m=0.02}$.

\section{CONCLUSIONS}

Tempering yields an integrated autocorrelation that is about a factor of three smaller than the HMC run, although much better data is needed to make this reliable! Tempering costs about three times more than the single HMC run at the smallest mass, so it is clearly faster if more than one mass is required, as is usually the case!

For realistic simulations, using improved ac- 
tions on large, smooth lattices at very small quark masses, tempered methods are very likely to be of benefit. This is especially true for Wilson fermions, where many $\kappa$ values are needed in order to extract meaningful physics.

\section{Acknowledgements}

This work was partially supported by HCMFellowship contract ERBCHBGCT940665, and currently the Japanese Society for the Promotion of Science. The author is grateful to both the Pisa and Tsukuba lattice groups for many useful discussions related to this work.

\section{REFERENCES}

1. G. Boyd et al., Proceedings, Lattice 96, IFUP-TH 47/96, hep-lat/96008123; B. Allés, et al., Phys. Lett B 389 (1996) 107; K. Bitar et al., Phys. Rev. D42 (1990) 3794.

2. E. Marinari and G. Parisi, Europhys. Lett. 19 (1992) 451; A. P. Lyubartsev et al., J. Chem. Phys. 96 (1992) 1776; L. Fernández et al., J. Phys. I France 5 (1995) 1247; E. Marinari, proceedings, Bielefeld workshop on Multi-scale Phenomena, October 1996. and cond-mat/9612010.

3. G. Boyd, proceedings, Bielefeld workshop on Multi-scale Phenomena, October 1996; heplat/9701009.

4. S. Gottlieb et al., Phys. Rev. D 35 (1987) 2531; A. D. Kennedy, Nucl. Phys. B (Proc. Suppl.) 30 (1993) 96.

5. See, for example, A. D. Sokal, in Quantum Fields on the Computer, Ed. M. Creutz, World Scientific 1992. 